\title{
Metric properties of generalized Cantor products
}

by

\author{
Y. LACROIX (Istres)
}

0. Introduction. Generalized Cantor products are algorithms that give a representation of real numbers $x \in[0,1$ [ as infinite products of rational ones. They have been developed in [Opp] first. Let us present those we shall consider from the metric point of view in this paper.

The letter $k$ shall denote an integer $\geq 1$. For any $x \in\left[0,1\left[\right.\right.$, let $r_{0}(x) \in \mathbb{N}$ and $T(x) \in[0,1[$ be defined by

$$
\frac{r_{0}(x)-1}{r_{0}(x)+k-1} \leq x<\frac{r_{0}(x)}{r_{0}(x)+k}, \quad T(x):=x\left(\frac{r_{0}(x)+k}{r_{0}(x)}\right) .
$$

One can see that $r_{0}(x)=[k x /(1-x)]+1$. Define, for any real number $z \geq 1$,

$$
\begin{aligned}
a_{z} & =(z-1) /(z+k-1), \\
b_{z} & =a_{z} / a_{z+1}=a_{(z-1)(z+k)+1}, \\
J_{z} & =\left[a_{z}, a_{z+1}[.\right.
\end{aligned}
$$

The sequences $\left(a_{n}\right)_{n \geq 1}$ and $\left(b_{n}\right)_{n \geq 1}$ are strictly increasing from 0 to 1 . By definitions we have $\bigcup_{n \geq 1} J_{n}=\left[0,1\left[, J_{n} \cap J_{m}=\emptyset\right.\right.$ if $n \neq m$ and $T(x)=x a_{n+1}^{-1}$ on $J_{n}$. Moreover,

$$
T\left(J_{n}\right)=\left[b_{n}, 1[.\right.
$$

Thus, according to the terminology of F. Schweiger (see [Sch]), the triple $\left(T,\left[0,1\left[,\left(J_{n}\right)_{n \geq 1}\right)\right.\right.$ is a measurable fibered system on $[0,1[$ with the Borel $\sigma$-algebra $B$.

Given $k \geq 1$ and $x \in\left[0,1\left[\right.\right.$, we define the sequence $\left(r_{t}(x)\right)_{t \geq 0}$ as follows:

$$
r_{t}(x)=r_{0}\left(T^{(t)}(x)\right),
$$

where $T^{(t)}$ denotes the $t$ th iterate of $T\left(T^{(0)}=\operatorname{Id}_{[0,1[}\right)$.

Research partially supported under DRET contract 901636/A000/DRET/DS/SR. 
W. Sierpiński ([Sie-1]) and A. Oppenheim ([Opp]) showed that for any integer $k \geq 1$ and any $x \in[0,1[$, with (3),

$$
x=\prod_{i=0}^{\infty} \frac{r_{i}(x)}{r_{i}(x)+k} .
$$

The case $k=1$ corresponds to Cantor's product (see [Per]). Generalizations of Cantor's product given in [Kn-Kn] do not overlap with those from [Sie-1] or $[\mathrm{Opp}]$, and do not arise from fibered systems on $[0,1[$.

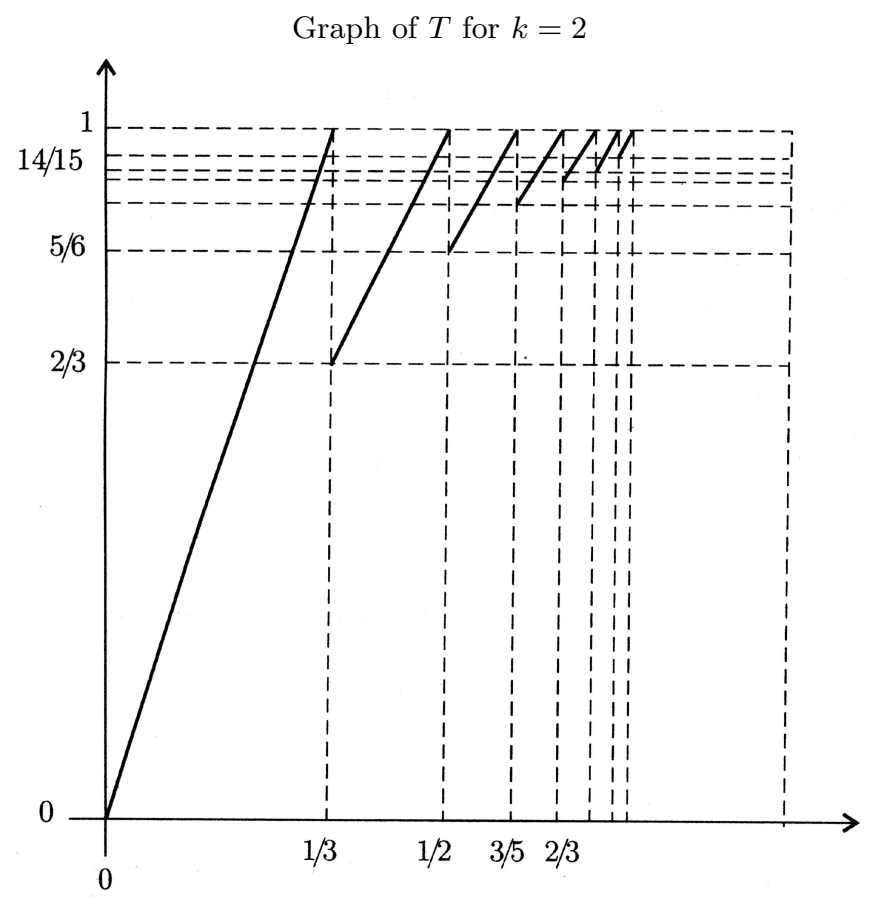

Euler's formula (see [MF-VP]) and Escott's formula ([Esc], [Sie-2])

$$
\sqrt{\frac{x-1}{x+1}}=\prod_{n=0}^{\infty} \frac{\varphi^{(n)}(x)}{\varphi^{(n)}(x)+1}, \quad \sqrt{\frac{x-2}{x+2}}=\prod_{n=0}^{\infty} \frac{\gamma^{(n)}(x-1)}{\gamma^{(n)}(x-1)+2},
$$

where $\varphi(x)=2 x^{2}-1$ and $\gamma(z)=z^{3}+3 z^{2}-2$, both give product expansions for integer $x$ (with $k=1$ or $k=2$ ). Some other formulas can be derived from the work of Ostrowski [Ost] (see also [MF-VP]). P. Stambul ([Sta]) points out the following Cantor product expansion

$$
\sqrt{2}-1=\prod_{n=0}^{\infty} \frac{\varphi^{(n)}(1)}{\varphi^{(n)}(1)+1},
$$

where $\varphi(x)=4 x^{2}-1+2 x \sqrt{2 x^{2}-1}$ is not a polynomial. Thus, quadratic 
irrationals in $[0,1$ [ are not characterized by the fact that their sequence of digits for the Cantor product has ultimately polynomial growth (cf. [Eng]).

In Section 1 we give some preliminary notations for cylinder sets and describe admissible sequences of digits $r_{n}(x)$ which occur in the product formula (4).

Our purpose is to study, as has been done for several other fibered systems (e.g. continued fractions in $[\mathrm{Khi}]$ ), the metric properties of the system $(T,[0,1[, B)$. The motivation for this is that in the case of continued fractions, the asymptotic behaviour for the relevant sequence of digits was deduced from the identification of the density $1 /(\log 2 \cdot(1+x))$ for a Lebesguecontinuous ergodic invariant measure on $[0,1]$, for the transformation

$$
x \mapsto \frac{1}{x}-\left[\frac{1}{x}\right] \quad \text { if } x \neq 0, \quad \text { and } \quad 0 \mapsto 0
$$

(see $[$ Khi] or $[\mathrm{Sch}]$ ).

But it appears, in Section 2, that the only probability invariant measure for $T$ is the Dirac measure at 0 , and that all $\sigma$-finite $\lambda$-continuous invariant measures for $T$ are determined by their restrictions to wandering sets for $T$. Therefore, it should be the case that $T$ is not ergodic with respect to $\lambda$.

However, in Section 3, in analogy with what happens in the case of Sylvester's series (see [Ver], [Sch]), and in some sense quite in contrast to what occurs for continued fractions, it appears that the limit function

$$
\beta(x)=\lim _{n \rightarrow \infty} \frac{\log r_{n}(x)}{2^{n}}
$$

exists $\lambda$-a.e., which enables us to conclude the nonergodicity of $T$ with respect to $\lambda$. The limit function $\beta$ should be proved to have most of the properties the relevant one for Sylvester's series was proved to have in [Go$\mathrm{Sm}$ ], where it essentially was providing the first explicitly defined function having jointly continuous occupation density (see also [Gal]).

Finally, in Section 4, we introduce the sequence of random variables $\left(t_{n}(\cdot)\right)_{n \geq 0}$ defined on $[0,1[$ by

$$
t_{n}(x)=\frac{T^{(n+1)}(x)-b_{r_{n}(x)}}{1-b_{r_{n}}(x)}, \quad x \in[0,1[, n \geq 0 .
$$

We show, using a modified version of a theorem of W. Philipp ([Phi]) in [Sch], Chapter 11, that $\lambda$-a.e., the sequence $\left(t_{n}(x)\right)_{n \geq 0}$ is completely uniformly distributed modulo 1 (see $[\mathrm{Ku}-\mathrm{Ni}]$ ). This generalizes some similar uniform distribution for Sylvester's series, or Engel's series, proved in [Sch-1].

The author would like to express his thanks to Professors J. P. Allouche, P. Liardet, F. Schweiger, B. Host, and to the referee, for valuable discussions or useful remarks. 
1. Admissible sequences of digits. From [Sie-1] and the definition of $T$ one has

$$
x=\prod_{i=0}^{\infty} \frac{r_{i}(x)}{r_{i}(x)+k}, \quad T^{(n+1)}(x) \in\left[b_{r_{n}}, 1[,\right.
$$

with

$$
T^{(n)}(x) \in\left[\frac{r_{n}-1}{r_{n}+k-1}, \frac{r_{n}}{r_{n}+k}\left[\quad \text { and } \quad r_{n}=r_{n}(x) .\right.\right.
$$

This will be called the $T$-expansion of $x$.

Take 1 as the value of the empty product, and let $n \geq 0$. One has

$$
\begin{aligned}
0 & <\prod_{j=0}^{n} \frac{r_{j}(x)}{r_{j}(x)+k}-x<\left(\prod_{j=0}^{n-1} \frac{r_{j}(x)}{r_{j}(x)+k}\right)\left(\frac{r_{n}(x)}{r_{n}(x)+k}-\frac{r_{n}(x)-1}{r_{n}(x)+k}\right) \\
& <\frac{k}{\left(r_{n}(x)+k\right)\left(r_{n}(x)+k-1\right)} .
\end{aligned}
$$

Let $n$ be an integer $\geq 1$ and let $r:=\left(r_{0}, \ldots, r_{n-1}\right) \in \mathbb{N}^{* n}$. The set

$$
B(r):=J_{r_{0}} \cap T^{-1}\left(J_{r_{1}}\right) \cap \ldots \cap T^{(-n+1)}\left(J_{r_{n-1}}\right)
$$

is said to be a cylinder set of rank $n$ if it is not empty. For $r=\left(r_{0}, \ldots, r_{n-1}\right)$ $\in \mathbb{N}^{n}$ (respectively $p=\left(p_{i}\right)_{i \geq 0}$ ) and $j \in[0, n]$ (resp. $j \geq 0$ ), define

$$
\Pi_{j}(r):=\prod_{i=0}^{j-1} \frac{r_{i}}{r_{i}+k} \quad\left(\operatorname{resp} . \Pi_{j}(p):=\prod_{i=0}^{j-1} \frac{p_{i}}{p_{i}+k}\right) .
$$

If $B(r)$ is a cylinder set of rank $n$ we easily get from (1), (2) and (5),

$$
B(r)=\left[\Pi_{n}(r) b_{r_{n-1}}, \Pi_{n}(r)[.\right.
$$

Definition 1.1. An $n$-uple $r=\left(r_{0}, \ldots, r_{n-1}\right)$ (resp. a sequence $p=$ $\left.\left(p_{m}\right)_{m \geq 0} \in \mathbb{N}^{\mathbb{N}}\right)$ is said to be a $T$-admissible $n$-uple (resp. sequence) of digits if $B(r) \neq \emptyset$ (resp. $B\left(p_{0}, \ldots, p_{n-1}\right) \neq \emptyset$ for all $\left.n \geq 1\right)$. The set of $T$-admissible $n$-uples will be denoted by $A_{n}$.

From (5), $p$ is a $T$-admissible sequence of digits if and only if for all $n \geq 0$, one has $\left[b_{p_{n}}, 1\left[\cap J_{p_{n+1}} \neq \emptyset\right.\right.$.

Proposition 1.1. A sequence $p=\left(p_{n}\right)_{n \geq 0}$ of natural numbers is a $T$ admissible sequence of digits if and only if for all $n \geq 0$ one has

$$
p_{n+1} \geq p_{n}^{2}+\left(p_{n}-1\right)(k-1)\left(\geq p_{n}^{2}\right) .
$$

Proof. Since $b_{r}$ has the form $a_{(r-1)(r+k)+1}$, an admissible sequence $\left(p_{n}\right)_{n \geq 0}$ is characterized by the inequalities $b_{p_{n}}<a_{p_{n+1}+1}, n \geq 0$. In other words,

$$
\frac{\left(p_{n}-1\right)\left(p_{n}+k\right)}{\left(p_{n}-1\right)\left(p_{n}+k\right)+k}<\frac{p_{n+1}}{p_{n+1}+k} .
$$

After simplification, we get the desired inequality. 
Remark 1.1. Let $p(\cdot)$ be the polynomial $p(x):=x^{2}+(x-1)(k-1)$. From (2) we have $a_{n}=a_{n+1} a_{p(n)}=a_{n+1} a_{p(n)+1} a_{p^{2}(n)}$. Hence by induction we obtain the following product formula:

$$
\frac{n-1}{n-1+k}=\prod_{j=1}^{\infty} \frac{p^{(j)}(n)}{p^{(j)}(n)+k} .
$$

According to Proposition 1.1, formula (8) gives the $T$-expansion of $(n-1) /(n-1+k)$ for $n \in \mathbb{N}$ (this was known from [Opp]). However, formula (8) holds for all real numbers $k \geq 1$ and $n \geq 1$.

2. Invariant measures. The transformation $T$ is such that $T(0)=0$ and if $x \in] 0,1\left[\right.$, the sequence $\left(T^{(n)}(x)\right)_{n \geq 0}$ is strictly increasing to 1 . Thus, from the Riesz representation theorem and the individual ergodic theorem, using Cesàro means, taking any generic point for $\mu$ if $\mu$ is an ergodic invariant probability measure, one can see that necessarily, for any $f \in \mathcal{C}(\mathbb{R} / \mathbb{Z})$, $\int f d \mu=\lim _{x \rightarrow 1^{-}} f(x)$ : since $T(0)=0$ is the only fixed point for $T$, one must have $\mu=\delta_{0}$, where $\delta_{0}$ denotes the Dirac measure at point 0 .

$\mathrm{R}$ e mark 2.1. It is more interesting to consider probability measures $\mu$ which are quasi-invariant under $T$, that is to say, $\mu$ is equivalent to $\mu \circ T^{-1}$. We give an example of such a measure which is discrete. Let $\beta_{j}, j \in \mathbb{Z}$, be the points in $[0,1[$ (identified with $\mathbb{R} / \mathbb{Z}$ ) given by

$$
\beta_{n}:=\frac{p^{(n)}(2)-1}{p^{(n)}(2)-1+k} \quad \text { and } \quad \beta_{-n}=(k+1)^{-n-1}
$$

for $n=0,1,2, \ldots$ By (5) and (8) one has

$$
T^{(n)}\left(\frac{1}{k+1}\right)=\prod_{j=0}^{\infty} \frac{p^{(j)}\left(p^{(n)}(2)\right)}{p^{(j)}\left(p^{(n)}(2)\right)+k} \quad \text { for } n \geq 0
$$

and

$$
T\left((k+1)^{-(m+1)}\right)=(k+1)^{-m} \quad \text { for } m \geq 1 .
$$

Hence $T\left(\beta_{n}\right)=\beta_{n+1}$ for all $n \in \mathbb{Z}$. Let $\delta_{a}$ denote the Dirac measure at $a$; then $\delta_{b_{n}} \circ T^{-1}=\delta_{b_{n+1}}$. This proves that the probability measure $\mu:=\frac{1}{3} \sum_{n \in \mathbb{Z}} 2^{-|n|} \delta_{\beta_{n}}$ is quasi-invariant under $T$.

Now let us look at $\sigma$-finite $\lambda$-continuous invariant measures. Let $U$ be any proper neighbourhood of 1 , e.g. take $U=[a, 1], 0<a<1$, and extend $T$ from $\left[0,1\left[\right.\right.$ to the 1 -torus $[0,1]$ setting $T(1)=1=0$. Let $V=T^{-1}(U) \backslash U$. Then define $V_{n}=T^{(n)}(V), n \in \mathbb{Z}$. It is a so called wandering set; indeed, using the fact that the sequence $\left(T^{(n)}(U)\right)_{n \in \mathbb{Z}}$ is decreasing, one has

$$
\bigcup_{n=-\infty}^{\infty} V_{n}=[0,1] \quad \text { and } \quad V_{n} \cap V_{m}=\emptyset \quad \text { for } m \neq n .
$$


Now assume we want to determine the density for a $\sigma$-finite $T$-invariant $\lambda$-continuous measure. Then if we take any positive, measurable and $\sigma$-finite function on $V$, we can define it on any $V_{n}$, taking its image via $T^{(n)}$, and finally we obtain a $\sigma$-finite density for a $T$-invariant $\lambda$-continuous measure (use (9)). For example, take $a=(k+2) / 2(k+1)$; then

$$
V=\left[\frac{k+2}{2(k+1)^{2}}, \frac{k+2}{2(k+1)}[\text {. }\right.
$$

3. Nonergodicity of $T$ with respect to $\lambda$, and asymptotic behaviour of $\left(r_{n}(x)\right)_{n \geq 0}$

Lemma 3.1. There are two positive constants $d_{1}$ and $d_{2}$ such that for any nonempty cylinder set $B\left(r_{0}, \ldots, r_{n-1}\right)$ of rank $n \geq 1$ and for any integers $w, j(w \geq j \geq 1)$ such that $B\left(r_{0}, \ldots, r_{n-1}, j, w\right)$ is a nonempty cylinder set of rank $n+2$ one has

$$
d_{1} \frac{j^{2}}{w^{2}} \leq \frac{\lambda\left(B\left(r_{0}, \ldots, r_{n-1}, j, w\right)\right)}{\lambda\left(B\left(r_{0}, \ldots, r_{n-1}, j\right)\right)} \leq d_{2} \frac{j^{2}}{w^{2}} .
$$

Proof. Put $B=B\left(r_{0}, \ldots, r_{n-1}, j, w\right), A=B\left(r_{0}, \ldots, r_{n-1}, j\right)$ and $P=$ $\Pi_{n}(r)$ for short, where $r=\left(r_{0}, \ldots, r_{n-1}\right)$ (cf. (6)). Then, with (7),

$$
\lambda(A)=P \frac{k}{(j+k)(j+k-1)}, \quad \lambda(B)=P \frac{j k}{(j+k)(w+k)(w+k-1)} .
$$

Therefore,

$$
\frac{\lambda(B)}{\lambda(A)}=\frac{j(j+k-1)}{(w+k)(w+k-1)},
$$

and the inequalities of the lemma follow with constants (for example) $d_{1}=$ $\left(k^{2}+k\right)^{-1}$ and $d_{2}=k$.

LEMmA 3.2. The limit function $\beta(x):=\lim _{n \rightarrow \infty}\left(\log r_{n}(x)\right) / 2^{n}$ exists $\lambda$ a.e. Moreover, $\beta(\cdot)$ is measurable and there exists a constant $\gamma>0$ such that for all $j \geq 1, n \geq 0$ and all $\varepsilon>0$ one has

$$
\left\{\begin{array}{r}
\lambda\left(\left\{x: r_{n}(x)=j \text { and } 0 \leq \beta(x)-2^{-n} \log j \leq \varepsilon\right\}\right) \\
\geq\left(1-\frac{2}{e^{\gamma \varepsilon 2^{n}}-1}\right) \lambda\left(\left\{r_{n}=j\right\}\right), \\
\beta(x)=\frac{1}{2}\left(\log r_{1}(x)+\sum_{n=0}^{\infty} \frac{\log \left(r_{n+1}(x) / r_{n}(x)^{2}\right)}{2^{n}}\right) \quad \lambda \text {-a.e. }
\end{array}\right.
$$

Proof. The second part of formula (10) is obvious, provided the $\lambda$-a.e. existence of the limit function $\beta$ is known.

Let $\varepsilon>0$ and for $x \in\left[0,1\left[\right.\right.$ define $\beta_{n}(x):=2^{-n} \log r_{n}(x)$. Since $r_{n+1}(x) \geq r_{n}(x)^{2}$, the sequence $\left(\beta_{n}(x)\right)_{n \geq 0}$ is not decreasing. Then $\beta_{n+1}(x)$ 
$-\beta_{n}(x)>\varepsilon$ is equivalent to $r_{n+1}(x)>\exp \left(\varepsilon 2^{n+1}\right) r_{n}(x)^{2}$. From Lemma 3.1, we get

$$
\lambda\left\{r_{n}=j \text { and } \beta_{n+1}-\beta_{n}>\varepsilon\right\} \leq d_{2}\left(\sum_{\substack{w \\ w>j^{2} \exp \left(\varepsilon 2^{n+1}\right)}} \frac{j^{2}}{w^{2}}\right) \lambda\left\{r_{n}=j\right\} .
$$

But it follows from elementary calculus that for all $j \geq 1$,

$$
\sum_{\substack{w \\ w>j^{2} \exp \left(\varepsilon 2^{n+1}\right)}} \frac{j^{2}}{w^{2}} \leq \frac{2}{e^{\varepsilon 2^{n+1}}} .
$$

Using (11) and (12), we obtain

$$
\lambda\left(\left\{r_{n}=j \text { and } \beta_{n+1}-\beta_{n}>\varepsilon\right\}\right) \leq 2 e^{-\varepsilon 2^{n+1}} \lambda\left(\left\{r_{n}=j\right\}\right) .
$$

Define $\eta_{m}=(\sqrt{2}-1)(\sqrt{2})^{-(m+1)}$, so that $\sum_{m>1} \eta_{m}=1$. Let $n \geq 0, m \geq 1$ be integers and assume $\beta_{n+s}(x)-\beta_{n+s-1}(x) \leq \varepsilon \eta_{s}$ for all $s \in\{1,2, \ldots, m\}$. Then $\beta_{n+m}(x)-\beta_{n}(x) \leq \varepsilon$ so that for

$$
X_{n}(j ; \varepsilon):=\left\{x: r_{n}(x)=j \text { and } \exists m \geq 1, \beta_{n+m}(x)-\beta_{n}(x)>\varepsilon\right\}
$$

we obtain

$$
\begin{array}{r}
\lambda\left(X_{n}(j ; \varepsilon)\right) \leq \lambda\left(\left\{r_{n}=j \text { and } \exists m \geq 1, \beta_{n+m}-\beta_{n+m-1}>\varepsilon \eta_{m}\right\}\right) \\
\quad \leq 2\left(\sum_{m \geq 1} e^{-\varepsilon \eta_{m} 2^{n+m+1}}\right) \lambda\left(\left\{r_{n}=j\right\}\right) \leq \frac{2}{e^{\gamma \varepsilon 2^{n}}-1} \lambda\left(\left\{r_{n}=j\right\}\right)
\end{array}
$$

where $\gamma=\sqrt{2}-1$. But (13) is nothing but inequality (10) of Lemma 3.2. If we sum over $j$ all inequalities (10) ( $n$ fixed) we also get

$$
\lambda\left(\left\{\beta-\beta_{n} \leq \varepsilon\right\}\right) \geq 1-\frac{2}{e^{\gamma \varepsilon 2^{n}}-1} .
$$

Now it is quite clear that the sequence $\left(\beta_{n}(x)\right)_{n \geq 0}$ converges (in $[0, \infty[)$ for almost all $x \in\left[0,1\right.$ [. Since $\beta_{n}$ is measurable, so is $\beta$.

R e mark 3.1. Notice that $\beta$ satisfies the following functional equations:

$$
\beta(T x)=2 \beta(x) \quad \text { and } \quad \beta\left(\frac{1}{k+1} x\right)=\frac{1}{2} \beta(x) .
$$

As in the case of Sylvester's series (see [Go-Sm]), it can be proved that $\beta$ is dense in its epigraph and has local minima at rational points exactly. In [Go-Sm] it was first proved that the $\beta$ function for Sylvester's series has a $\mathcal{C}^{\infty}$ density. In [Gal], it was proved that for the Cantor product, $\beta$ has a $\mathcal{C}^{1}$ density. This last result at least should hold for the generalized Cantor products we are dealing with here.

THEOREM 3.1. $T$ is not ergodic with respect to $\lambda$, i.e. there exist two disjoint T-invariant subsets of $[0,1[$ with positive Lebesgue measure. 
Proof. Let $J$ be a nonempty open subinterval of $] 0, \infty[$. Choose $\varepsilon>0$ such that there exist integers $p \geq 1$ and $m \geq 1$ satisfying

$$
\left[\frac{\log p}{2^{m}}-\varepsilon, \frac{\log p}{2^{m}}+\varepsilon\right] \subset J
$$

Let $N_{\varepsilon}$ be an integer such that $1-2 /\left(e^{\gamma \varepsilon 2^{n}}-1\right)>0$ for all $n \geq N_{\varepsilon}$. We can easily choose integers $d \geq 2$ and $n \geq N_{\varepsilon}$ in order to have $2^{-n} \log d$ close enough to $2^{-m} \log p$ such that we still have

$$
\left[\frac{\log d}{2^{n}}-\varepsilon, \frac{\log d}{2^{n}}+\varepsilon\right] \subset J
$$

Since $\lambda\left(\left\{r_{n}=d\right\}\right)>0$ for any integer $d \geq 1$, inequality (10) implies $\lambda(\{x$ : $\beta(x) \in J\})>0$ and the set

$$
E(J):=\left\{x: \beta(x) \in \bigcup_{m \in \mathbb{Z}} 2^{m} J\right\}
$$

is measurable and $T$-invariant with $\lambda(E(J))>0$. Let $J$ and $J^{\prime}$ be two nonempty open intervals such that $J \subset\left[\frac{1}{2}, \frac{3}{4}\right.$ [ and $J^{\prime} \subset\left[\frac{3}{4}, 1[\right.$. Then the sets $E(J)$ and $E\left(J^{\prime}\right)$ are disjoint, $T$-invariant and $\mu(E(J))>0$ and $\mu\left(E\left(J^{\prime}\right)\right)>0$. This ends the proof.

4. Uniform distribution. In this section we study the distribution of $T^{(n)}(x)$ in the interval $\left[a_{r_{n}(x)}, a_{r_{n}(x)+1}\left[\right.\right.$. More precisely, let $\left(t_{n}(\cdot)\right)_{n \geq 0}$ be the sequence of random variables defined on $[0,1[$ by

$$
t_{n}(x):=\frac{T^{(n)}(x)-a_{r_{n}}}{a_{r_{n}+1}-a_{r_{n}}}=\frac{T^{(n+1)}(x)-b_{r_{n}(x)}}{1-b_{r_{n}(x)}}, \quad x \in[0,1[, n \geq 0 .
$$

Let $\Phi_{n}(\cdot)$ denote the distribution function of $t_{n}(\cdot)$, and define

$$
W_{n}(d):=\left\{x: 0 \leq t_{n}(x)<d\right\}, \quad d \in[0,1] .
$$

THEOREM 4.1. The sequence of random variables $\left(t_{n}(\cdot)\right)_{n \geq 0}$ is identically and uniformly distributed (i.e., $\Phi_{n}(d)=d$ for $0 \leq d \leq 1, n \geq 0$ ).

Proof. For $d \in[0,1]$ we have $\Phi_{n}(d)=\lambda\left(\left\{x: 0 \leq t_{n}(x)<d\right\}\right)$. Let $r=\left(r_{0}, \ldots, r_{n}\right) \in A_{n+1}$ (see Definition 1.1). Since $T^{(n+1)}(x)=\Pi_{n+1}^{-1}(r) x$ on $B\left(r_{0}, \ldots, r_{n}\right)$ and $T^{(n+1)}(B(r))=\left[b_{r_{n}}, 1\left[\right.\right.$, the set $W_{n}(d)$ is the union of the following pairwise disjoint sets:

$$
B(r) \cap W_{n}(d)=\left\{x: b_{r_{n}} \Pi_{n+1}(r) \leq x<\Pi_{n+1}(r)\left(b_{r_{n}}+d\left(1-b_{r_{n}}\right)\right)\right\} .
$$

But $\lambda\left(B(r) \cap W_{n}(d)\right)=d \lambda(B(r))$ so

$$
\lambda\left(W_{n}(d)\right)=\sum_{r \in A_{n+1}} d \lambda(B(r))=d .
$$


With a view to the study of the $\lambda$-a.e. complete uniform distribution of the sequence $\left(t_{n}(x)\right)_{n \geq 0}$, let us introduce

Definition 4.1. Let $p \in \mathbb{N}$ and $\left(d_{0}, \ldots, d_{p}\right),\left(d_{0}^{\prime}, \ldots, d_{p}^{\prime}\right) \in[0,1]^{p+1}$. Then, for any $n \geq 0$, let

$$
E_{n}\left(d_{0}, \ldots, d_{p}\right)=W_{n}\left(d_{0}\right) \cap \ldots \cap W_{n+p}\left(d_{p}\right) .
$$

If $m \geq 1$, let

$$
\left(d_{0}, \ldots, d_{p}, 1^{m}, d_{0}^{\prime}, \ldots, d_{p}^{\prime}\right)=(d_{0}, \ldots, d_{p}, \underbrace{1, \ldots, 1}_{m \text { times }}, d_{0}^{\prime}, \ldots, d_{p}^{\prime}) .
$$

Let $d_{-1}=1$ and $E_{n}(\emptyset)=[0,1]$.

With the above notations, we have

TheOREM 4.2. For any integer $p \geq 0$, for any integer $n \geq 1$, any integer $m \geq 0$, any $\left(d_{0}, \ldots, d_{p}, d_{0}^{\prime}, \ldots, d_{p}^{\prime}\right) \in[0,1]^{2(p+1)}$,

$$
\begin{gathered}
\left|\lambda\left(E_{n}\left(d_{0}, \ldots, d_{p}, 1^{m}, d_{0}^{\prime}, \ldots, d_{p}^{\prime}\right)\right)-d_{0} \ldots d_{p} d_{0}^{\prime} \ldots d_{p}^{\prime}\right| \\
\leq 20(p+1)^{2} k^{2}(k+1)^{2}\left(\frac{1}{2}\right)^{n}, \\
\left|\lambda\left(E_{n}\left(d_{0}, 1^{m}, d_{0}^{\prime}\right)\right)-d_{0} d_{0}^{\prime}\right| \leq \frac{5}{2} k^{2}(k+1)^{2}\left(\frac{1}{2}\right)^{n+m} .
\end{gathered}
$$

Proof. Step 1. We need several lemmas and definitions.

Lemma 4.1. For any $n \in \mathbb{N}, m \geq 1, r=\left(r_{0}, r_{1}, \ldots, r_{n+m}\right) \in A_{n+m+1}$, one has

$$
\begin{aligned}
\frac{r_{n}^{2} \lambda\left(B\left(r_{n+1}, \ldots, r_{n+m}\right)\right)}{k} & \leq \frac{\lambda(B(r))}{\lambda\left(B\left(r_{0}, \ldots, r_{n}\right)\right)} \\
& \leq(k+1) r_{n}^{2} \lambda\left(B\left(r_{n+1}, \ldots, r_{n+m}\right)\right) \\
& \leq \frac{k(k+1)}{2^{m}}
\end{aligned}
$$

Moreover,

$$
\lambda\left(B\left(r_{0}, \ldots, r_{n}\right)\right) \leq \min \left\{2^{-(n+1)}, \frac{k}{\left(r_{n}+k\right)\left(r_{n}+k-1\right)}\right\} .
$$

Proof. Notice that

$$
\begin{aligned}
\lambda(B(r)) & =\left(\frac{r_{0}}{r_{0}+k} \cdots \frac{r_{n+m-1}}{r_{n+m-1}+k}\right) \frac{k}{\left(r_{n+m}+k\right)\left(r_{n+m}+k-1\right)} \\
& =\lambda\left(B\left(r_{0}, \ldots, r_{n}\right)\right) \frac{\left(r_{n}+k\right)\left(r_{n}+k-1\right)}{k} \lambda\left(B\left(r_{n+1}, \ldots, r_{n+m}\right)\right)
\end{aligned}
$$

and then inequality (14) follows from

$$
\frac{x^{2}}{k} \leq \frac{(x+k)(x+k-1)}{k} \leq(k+1) x^{2} \quad \text { for } x \geq 1
$$


On the other hand, put $p(x)=x^{2}+(x-1)(k-1)$ and assume that $r_{s-1}=1$ $\left(\neq r_{s}\right)$ for a digit with $0<s \leq n$. Proposition 1.1 and (7) imply

$$
\lambda\left(B\left(r_{0}, \ldots, r_{n}\right)\right) \leq(k+1)^{-s} \frac{k}{\left(p^{(n-s)}\left(r_{s}\right)+k-1\right)\left(p^{(n-s)}\left(r_{s}\right)+k\right)} .
$$

If $r_{s}=1=r_{n}$ the inequality (15) is evident. Otherwise $r_{s} \geq 2$ but $p^{(n-s)}(2) \geq 2^{2^{n-s}}$ and therefore (16) is still true. It remains to prove (15). If $r_{n}=1$, the inequality follows from (16), otherwise we have

$$
\lambda\left(B\left(r_{n+1}, \ldots, r_{n+m}\right)\right) \leq k\left(p^{(m)}\left(r_{n}\right)\right)^{-2} \leq k r_{n}^{-2^{m+1}} \leq k r_{n}^{-2} 2^{-m} .
$$

Lemma 4.2. For positive natural numbers $n$ and $m$ let

$$
F_{n}(m)=\#\left\{\left(r_{0}, \ldots, r_{n-2}\right) \in \mathbb{N}^{n-1}:\left(r_{0}, \ldots, r_{n-2}, m\right) \in A_{n}\right\} .
$$

Then $F_{n}(m) \leq m$.

Proof. We use induction on $n$. It is clear that $F_{1}(m) \leq m$. Now, let $n \geq 1$ be given and assume $F_{n}(m) \leq m$ for all $m \geq 1$. Proposition 1.1 implies that for any $\left(r_{0}, \ldots, r_{n-1}, m\right) \in A_{n+1}$ one has $r_{n-1} \leq \sqrt{m}$. Therefore

$$
F_{n+1}(m) \leq \sum_{1 \leq j \leq \sqrt{m}} j \leq m
$$

Lemma 4.3. For any positive natural numbers $n, m$ and for any map $s: A_{n} \rightarrow \mathbb{N}^{m}$ satisfying $\left(\left(r_{0}, \ldots, r_{n-1}\right), s\left(r_{0}, \ldots, r_{n-1}\right)\right) \in A_{n+m}$, one has

$$
\sum_{r \in A_{n}} \lambda(B(r, s(r))) \leq \frac{5 k^{3}(k+1)^{3}}{2^{n+m}}
$$

(we identify $\mathbb{N}^{n+m}$ with $\mathbb{N}^{n} \times \mathbb{N}^{m}$ ).

P r o of. We first study the case $m=1$. If $n=1$, first notice that for any map $s_{1}: A_{1}=\mathbb{N}^{*} \rightarrow \mathbb{N}^{*}$ such that for any $r \in \mathbb{N}^{*},\left(r, s_{1}(r)\right) \in A_{2}$, from (7) and Proposition 1.1,

$$
\begin{aligned}
\sum_{r \in \mathbb{N}^{*}} \lambda\left(B\left(r, s_{1}(r)\right)\right) & \leq \sum_{r \geq 1} \frac{k r}{(r+k)\left(s_{1}(r)+k\right)\left(s_{1}(r)+k-1\right)} \\
& \leq \sum_{r \geq 1} \frac{k r}{(r+k)\left(r^{2}+(k-1) r+1\right)\left(r^{2}+(k-1) r\right)}
\end{aligned}
$$

But since $k \geq 1$,

$$
\sum_{r \geq 1} \frac{k}{(r+k)\left(r^{2}+(k-1) r+1\right)(r+k-1)} \leq \sum_{r \geq 1} \frac{1}{(r+1)\left(r^{2}+1\right)} \leq \frac{1}{2},
$$

and indeed $2 \leq 5 k^{3}(k+1)^{3}$. 
Assume now that $n \geq 2$. Then from Lemma 4.1, it follows that for any $r \in A_{n}, r=\left(r_{0}, \ldots, r_{n-1}\right)$,

$$
\lambda(B(r, s(r))) \leq k(k+1) \lambda(B(r)) \frac{r_{n-1}^{2}}{p\left(r_{n-1}\right)^{2}} \leq k(k+1) \frac{\lambda(B(r))}{r_{n-1}^{2}} \leq \frac{k^{2}(k+1)}{r_{n-1}^{4}} .
$$

Then, for any $N \geq 1$,

$$
\begin{gathered}
\sum_{r \in A_{n}} \lambda(B(r, s(r))) \leq k^{2}(k+1) \sum_{\substack{r \in A_{n} \\
r_{n-1}>N}} \frac{1}{r_{n-1}^{4}}+\sum_{\substack{r \in A_{n} \\
r_{n-1} \leq N}} \lambda(B(r, s(r))) \\
\leq k^{2}(k+1) \sum_{t>N} \frac{1}{t^{3}}+k(k+1) \sum_{\substack{r \in A_{n} \\
r_{n-1} \leq N}} \frac{\lambda(B(r))}{r_{n-1}^{2}} \\
\leq \frac{k^{2}(k+1)}{2 N^{2}}+k^{2}(k+1)^{2} \sum_{\substack{\left(r_{0}, \ldots, r_{n-1}\right) \in A_{n} \\
r_{n-1} \leq N}} \lambda\left(B\left(r_{0}, \ldots, r_{n-2}\right)\right) \frac{r_{n-2}^{2}}{r_{n-1}^{4}} .
\end{gathered}
$$

But $r_{n-1} \geq r_{n-2}^{2}$ and therefore with $g=4 k^{2}(k+1)^{2}$ and (16),

$$
\begin{aligned}
\sum_{r \in A_{n}} \lambda(B(r, s(r))) & \leq \frac{k^{2}(k+1)}{2 N^{2}}+\frac{g}{2^{n+1}} \sum_{\substack{\left(r_{0}, \ldots, r_{n-2}\right) \in A_{n-1} \\
r_{n-2} \leq \sqrt{N}}} r_{n-2}^{-6} \\
& \leq \frac{k^{2}(k+1)}{2 N^{2}}+\frac{g}{2^{n+1}} \sum_{1 \leq k \leq \sqrt{N}} k^{-5} .
\end{aligned}
$$

Passing to the limit as $N$ tends to infinity, we get the case $m=1$ with $\frac{5}{4} g$. The general case follows from (15) which gives

$$
\lambda(B(r, s(r))) \leq \lambda\left(B\left(r, s_{1}(r)\right)\right) \frac{k(k+1)}{2^{m-1}} .
$$

Definition 4.2. Let $n \geq 1$ be an integer. Let $r=\left(r_{0}, \ldots, r_{n-1}\right) \in A_{n}$. Let $d \in\left[0,1\left[\right.\right.$. Then define $r^{\prime}(d, r)$ to be the unique integer such that, if $r^{\prime \prime}=\left(r_{0}, \ldots, r_{n-1}, r^{\prime}(d, r)\right)$, we have

$$
\Pi_{n}(r)\left(b_{r_{n-1}}+d\left(1-b_{r_{n-1}}\right)\right) \in B\left(r^{\prime \prime}\right) .
$$

Denote the above admissible $(n+1)$-uple $r^{\prime \prime}$ by $r r^{\prime}(d, r)$ (as a concatenation). If $\left(r, r^{\prime}\right) \in \mathbb{N}^{n} \times \mathbb{N}^{m}$, let $r r^{\prime}$ be the $(n+m)$-uple defined by $r r^{\prime}=\left(r_{0}, \ldots, r_{n-1}, r_{0}^{\prime}, \ldots, r_{m-1}^{\prime}\right)$. Endow the sets $A_{n}$ with the lexicographic order. If $d=1$ and $r \in A_{n}$, let $r^{\prime}(1, r)=+\infty$, and $B(r,+\infty)=\emptyset$.

Let $n \geq 0$ and $m \geq 1$. Let $r \in A_{n+1}, r=\left(r_{0}, \ldots, r_{n}\right)$, and define

$$
A_{n+1, m}(r):=\left\{r^{\prime}=\left(r_{n+1}^{\prime}, \ldots, r_{n+m}^{\prime}\right) \in \mathbb{N}^{m}: r r^{\prime} \in A_{n+m+1}\right\} .
$$


Lemma 4.4. For any $q \geq 1$ and any $k \geq 1$,

$$
\begin{aligned}
& \frac{1}{(q+k)(q+k-1)} \\
& \quad>2\left(\sum_{m \geq 0} \frac{1}{(q+m+k)\left((q+m)^{2}+(q+m)(k-1)+1\right)(q+m+k-1)}\right) .
\end{aligned}
$$

Proof. The sum of the series is clearly bounded by

$$
\begin{aligned}
& \frac{1}{(q+k)(q+k-1)\left(q^{2}+q(k-1)+1\right)} \\
& \quad+\left(\sum_{t \geq q+1} \frac{1}{(t+k)(t+k-1)}\right) \frac{1}{(q+1)^{2}+(q+1)(k-1)+1} \\
& \leq \frac{1}{(q+k)(q+k-1)}\left(\frac{1}{(q+1)(q+k)-2 q-k+1}+\frac{1}{q+1}-\frac{1}{(q+1)(q+k)}\right) \\
& \leq\left(\frac{1}{q+1}\right) \frac{1}{(q+k)(q+k-1)},
\end{aligned}
$$

and $q \geq 1$.

S tep 2. Let $p^{\prime} \geq 1$. Using refining partitions of cylinders on $[0,1[$, one can see quite easily, with the use of Theorem 4.1 and Definition 4.2, that, given $\left(d_{0}, \ldots, d_{p^{\prime}}\right) \in[0,1]^{p^{\prime}+1}, n \geq 1$ and $r=\left(r_{0}, \ldots, r_{n}\right) \in A_{n+1}$,

$$
\sum_{\substack{r_{n+1} \in A_{n+1,1}(r) \\
r_{n+1}<r^{\prime}\left(d_{0}, r\right)}}^{\lambda\left(E_{n}\left(d_{0}, \ldots, d_{p^{\prime}}\right) \cap B(r)\right)=}\left(\sum _ { \substack { r _ { n + 2 } \in A _ { n + 2 , 1 } ( r r _ { n + 1 } ) \\
r _ { n + 2 } < r ^ { \prime } ( d _ { 1 } , r r _ { n + 1 } ) } } \cdots \left(\sum_{\left.\begin{array}{c}
r_{n+p^{\prime}} \in A_{n+p^{\prime}, 1}\left(r r_{n+1} \ldots r_{n+p^{\prime}-1}\right) \\
r_{n+p^{\prime}}<r^{\prime}\left(d_{p^{\prime}-1}, r r_{n+1} \ldots r_{n+p^{\prime}-1}\right)
\end{array}\right)}\right.\right.
$$

$$
\begin{aligned}
& +\sum_{\substack{r_{n+1} \in A_{n+1,1}(r) \\
r_{n+1}<r^{\prime}\left(d_{0}, r\right)}}\left(\ldots \left(\sum_{\substack{r_{n+p^{\prime}-1} \in A_{n+p^{\prime}-1,1}\left(r \ldots r_{n+p^{\prime}-2}\right) \\
r_{n+p^{\prime}-1}<r^{\prime}\left(d_{p^{\prime}-2}, r \ldots r_{n+p^{\prime}-2}\right)}}\right.\right. \\
& \left.\left.\lambda\left(B\left(r \ldots r_{n+p^{\prime}-1} r^{\prime}\left(d_{p^{\prime}-1}, r \ldots r_{n+p^{\prime}-1}\right)\right) \cap E_{n}\left(d_{0}, \ldots, d_{p^{\prime}}\right)\right)\right)\right)
\end{aligned}
$$

$+\ldots$

$+\sum_{\substack{r_{n+1} \in A_{n+1,1}(r) \\ r_{n+1}<r^{\prime}\left(d_{0}, r\right)}} \lambda\left(B\left(r r_{n+1} r^{\prime}\left(d_{1}, r r_{n+1}\right)\right) \cap E_{n}\left(d_{0}, \ldots, d_{p^{\prime}}\right)\right)$

$+\lambda\left(B\left(r r^{\prime}\left(d_{0}, r\right)\right) \cap E_{n}\left(d_{0}, \ldots, d_{p^{\prime}}\right)\right)$.

Let, for $i \in[1, p]$,

$$
X_{i}\left(d_{0}, \ldots, d_{p}, n\right)=\left|\lambda\left(E_{n}\left(d_{0}, \ldots, d_{i}\right)\right)-d_{i} \lambda\left(E_{n}\left(d_{0}, \ldots, d_{i-1}\right)\right)\right| .
$$


Notice that $X_{i}\left(d_{0}, \ldots, d_{p}, n\right)=0$ if $p=0$ or $d_{i} \in\{0,1\}$. Let, for $i \in[1, p]$,

$$
\begin{array}{r}
Y_{i}\left(d_{0}, \ldots, d_{p}, n\right)=\sum_{r \in A_{n+1}}\left(\ldots \left(\sum_{\begin{array}{c}
r_{n+i} \in A_{n+i, 1}\left(r r_{n+1} \ldots r_{n+i-1}\right) \\
r_{n+i}<r^{\prime}\left(d_{i-1}, r r_{n+1} \ldots r_{n+i-1}\right)
\end{array}}\left(\mathrm{r}_{n+1}, \ldots\right),\right.\right. \\
\left.\left.\lambda\left(B\left(r r_{n+1} \ldots r_{n+i} r^{\prime}\left(d_{i}, r \ldots r_{n+i}\right)\right) \cap E_{n}\left(d_{0}, \ldots, d_{p}\right)\right)\right) \ldots\right)[
\end{array}
$$

and

$$
Y_{0}\left(d_{0}, \ldots, d_{p}, n\right)=\sum_{r \in A_{n+1}} \lambda\left(B\left(r r^{\prime}\left(d_{0}, r\right)\right) \cap E_{n}\left(d_{0}, \ldots, d_{p}\right)\right) .
$$

Definition 4.3. Let $r^{\prime}(r)$ denote the smallest element of $A_{n, 1}(r)$ for $r \in A_{n}$.

Let, for $i \in \mathbb{N}^{*}$, with Definitions 4.2 and 4.3,

$$
\begin{aligned}
R_{i}(n)=\sum_{r \in A_{n+1}}\left(\ldots \left(\sum_{r_{n+i} \in A_{n+i, 1}\left(r r_{n+1} \ldots r_{n+i-1}\right)}\right.\right. \\
\left.\left.\lambda\left(B\left(r r_{n+1} \ldots r_{n+i} r^{\prime}\left(r \ldots r_{n+i}\right)\right)\right)\right) \ldots\right),
\end{aligned}
$$

and

$$
R_{0}(n)=\sum_{r \in A_{n+1}} \lambda\left(B\left(r r^{\prime}\left(d_{0}, r\right)\right)\right) .
$$

Define, for $i \in[1, p]$,

$$
\begin{aligned}
Z_{i}\left(d_{0}, \ldots, d_{p}, n\right)=\sum_{r \in A_{n+1}}\left(\ldots \left(\sum_{\left.\begin{array}{c}
r_{n+i} \in A_{n+i, 1}\left(r r_{n} \ldots r_{n+i-1}\right) \\
r_{n+i}<r^{\prime}\left(d_{i-1}, r r_{n} \ldots r_{n+i-1}\right)
\end{array}\right)}\right.\right. \\
\left.\left.\lambda\left(B\left(r r_{n} \ldots r_{n+i} r^{\prime}\left(d_{i}, r \ldots r_{n+i}\right)\right)\right)\right) \ldots\right),
\end{aligned}
$$

and

$$
Z_{0}\left(d_{0}, \ldots, d_{p}\right)=\sum_{r \in A_{n+1}} \lambda\left(B\left(r r^{\prime}\left(d_{0}, r\right)\right)\right)
$$

Observe that if $p>0$,

$$
\mid \sum_{r \in A_{n+1}}\left(\sum _ { \substack { r _ { n + 1 } \in A _ { n + 1 , 1 } ( r ) \\ r _ { n + 1 } < r ^ { \prime } ( d _ { 0 } , r ) } } \left(\ldots \sum_{\substack{r_{n+p} \in A_{n+p, 1}\left(r r_{n+1} \ldots r_{n+p-1}\right) \\ r_{n+p}<r^{\prime}\left(d_{p-1}, r r_{n+1} \ldots r_{n+p-1}\right)}}\right.\right.
$$$$
\begin{array}{r}
-d_{p-1}\left(\sum _ { r \in A _ { n + 1 } } \left(\sum _ { \substack { r _ { n + 1 } \in A _ { n + 1 , 1 } ( r ) \\
r _ { n + 1 } < r ^ { \prime } ( d _ { 0 } , r ) } } \left(\ldots \sum_{\begin{array}{c}
r_{n+p-1} \in A_{n+p-1,1}\left(r r_{n+1} \ldots r_{n+p-2}\right) \\
r_{n+p}<r^{\prime}\left(d_{p-2}, r r_{n+1} \ldots r_{n+p-2}\right)
\end{array}}\right.\right.\right. \\
\left.\left.\left.\lambda\left(B\left(r r_{n+1} \ldots r_{n+p-1}\right)\right)\right)\right)\right) \mid \leq Z_{p-1}\left(d_{0}, \ldots, d_{p}, n\right) .
\end{array}
$$ 
Then, from relations (17) to $(22)$, if we put $Z_{-1}\left(d_{0}, n\right)=0$,

$$
\begin{aligned}
& \left|\lambda\left(E_{n}\left(d_{0}, \ldots, d_{p}\right)\right)-d_{p} \lambda\left(E_{n}\left(d_{0}, \ldots, d_{p-1}\right)\right)\right| \\
& \leq \delta_{p \neq 0} \delta_{d_{p} \notin\{0,1\}}\left(2\left(\sum_{i=0}^{p-1} Y_{i}\left(d_{0}, \ldots, d_{p}, n\right)\right)+Z_{p-1}\left(d_{0}, \ldots, d_{p}, n\right)\right) \\
& \leq \underbrace{\delta_{p \neq 0} \delta_{d_{p} \notin\{0,1\}}\left(2\left(\sum_{i=0}^{p-1} R_{i}(n)\right)+Z_{p-1}\left(d_{0}, \ldots, d_{p}, n\right)\right)}_{W\left(d_{0}, \ldots, d_{p}, n\right)},
\end{aligned}
$$

where if $P$ is a proposition, $\delta_{P}=0$ if $P$ is false, 1 otherwise. Let

$$
\left(d_{0}, \ldots, d_{p}, 1^{m}, d_{0}^{\prime}, \ldots, d_{p}^{\prime}\right)=\left(a_{0}, \ldots, a_{2 p+m+1}\right) .
$$

From (17), (18), Definition 4.2 and repeated application of the triangle inequality,

$$
\begin{aligned}
& \left|\lambda\left(E_{n}\left(d_{0}, \ldots, d_{p}, 1^{m}, d_{0}^{\prime}, \ldots, d_{p}^{\prime}\right)\right)-d_{0} \ldots d_{p} d_{0}^{\prime} \ldots d_{p}^{\prime}\right| \\
& \quad \leq \sum_{i=1}^{p} X_{i}\left(d_{0}, \ldots, d_{p}, n\right)+\sum_{i=p+m+1}^{2 p+m+1} X_{i}\left(a_{0}, \ldots, a_{2 p+m+1}, n\right) .
\end{aligned}
$$

From Proposition 1.1, Definition 4.3, for any integer $m \geq 1$ and any $r \in A_{m}$,

$$
\begin{aligned}
& \sum_{p \geq r^{\prime}(r)} \lambda\left(B\left(r p r^{\prime}(r p)\right)\right) \\
& \quad \leq \sum_{p \geq r^{\prime}(r)}\left(\prod_{i=0}^{m-1} \frac{r_{i}}{r_{i}+k}\right) \frac{k p}{(p+k)\left(p^{2}+(k-1) p+1\right)\left(p^{2}+(k-1) p\right)},
\end{aligned}
$$

and from Lemma 4.4, with $q=r^{\prime}(r)$, we deduce from the above inequality that

$$
\sum_{p \geq r^{\prime}(r)} \lambda\left(B\left(r p r^{\prime}(r p)\right)\right) \leq \frac{1}{2} \lambda\left(B\left(r r^{\prime}(r)\right)\right) .
$$

Then, from definitions (20), (20)' and the above,

$$
R_{i}(n) \leq \frac{1}{2} R_{i-1}(n) \leq \ldots \leq\left(\frac{1}{2}\right)^{i} R_{0}(n) .
$$

It follows from (20), (21) and (23)-(25) that

$$
\begin{aligned}
\mid \lambda\left(E _ { n } \left(d_{0}, \ldots,\right.\right. & \left.\left.d_{p}, 1^{m}, d_{0}^{\prime}, \ldots, d_{p}^{\prime}\right)\right)-d_{0} \ldots d_{p} d_{0}^{\prime} \ldots d_{p}^{\prime} \mid \\
& \leq \sum_{i=1}^{p} W\left(d_{0}, \ldots, d_{i}, n\right)+\sum_{i=p+m+1}^{2 p+m+1} W\left(a_{0}, \ldots, a_{i}, n\right) \\
& \leq 4 p(p+1) R_{0}(n)+2(p+1)^{2} R_{p+m+1}(n) .
\end{aligned}
$$


From Lemma 4.3, we have

$$
R_{0}(n) \leq \frac{5 k^{2}(k+1)^{2}}{2^{n+1}}
$$

Thus, from (25), (26), (27), we obtain

$$
\begin{aligned}
& \left|\lambda\left(E_{n}\left(d_{0}, \ldots, d_{p}, 1^{m}, d_{0}^{\prime}, \ldots, d_{p}^{\prime}\right)\right)-d_{0} \ldots d_{p} d_{0}^{\prime} \ldots d_{p}^{\prime}\right| \\
& \quad \leq 10(p+1) k^{2}(k+1)^{2}\left(\frac{p}{2^{n}}+(p+1)\left(\frac{1}{2}\right)^{p+2}\left(\frac{1}{2}\right)^{n+m}\right),
\end{aligned}
$$

hence

$$
\begin{aligned}
\mid \lambda\left(E_{n}\left(d_{0}, \ldots, d_{p}, 1^{m}, d_{0}^{\prime}, \ldots, d_{p}^{\prime}\right)\right)- & d_{0} \ldots d_{p} d_{0}^{\prime} \ldots d_{p}^{\prime} \mid \\
\leq & 20(p+1)^{2} k^{2}(k+1)^{2}\left(\frac{1}{2}\right)^{n} .
\end{aligned}
$$

Now formula $(\alpha)$ of Theorem 4.2 is given in $(28)^{\prime}$ above, and $(\beta)$ comes from (28) in the case $p=0$. This ends the proof of Theorem 4.2.

TheOrem 4.3. For almost all $x$, the sequence $\left(t_{n}(x)\right)_{n \geq 0}$ is completely uniformly distributed in $[0,1]$, e.g., for almost all $x \in[0,1[$ and every $p \geq$ 0 , the sequence $\left(t_{n}(x), \ldots, t_{n+p}(x)\right)_{n \geq 0}$ is uniformly distributed in $[0,1]^{p+1}$. More precisely, for all $\varepsilon>0$ and all $\left(d_{0}, d_{1}, \ldots, d_{p}\right) \in[0,1]^{p+1}$, one has

$$
\begin{aligned}
\frac{1}{N} \sum_{n<N} \mathbf{1}_{\left[0, d_{0}\left[\times \ldots \times\left[0, d_{p}[\right.\right.\right.}\left(t_{n}(x), \ldots, t_{n+p}(x)\right) & \\
& =d_{0} d_{1} \ldots d_{p}+O\left(\frac{(\log N)^{3 / 2+\varepsilon}}{\sqrt{N}}\right), \quad \lambda \text {-a.e. }
\end{aligned}
$$

Proof. It is a direct application of Theorem $4.2(\alpha)$ and Theorem 11.3 from [Sch]. Indeed, given $p \geq 0$ and $\left(d_{0}, \ldots, d_{p}\right) \in[0,1]^{p+1}$ from $(\alpha)$, one has, if we let $E_{n}:=E_{n}\left(d_{0}, \ldots, d_{p}\right)$,

$$
\lambda\left(E_{n}\right)=d_{0} \ldots d_{p}+O\left(1 / 2^{n}\right),
$$

where the constant in the $O$ is bounded when $\left(d_{0}, \ldots, d_{p}\right)$ is fixed, and $E_{n}\left(d_{0}, \ldots, d_{p}\right) \cap E_{n+m+p+1}\left(d_{0}, \ldots, d_{p}\right)=E_{n}\left(d_{0}, \ldots, d_{p}, 1^{m}, d_{0}, \ldots, d_{p}\right)$, for $m$ large enough. Thus, we can find a convergent series of nonnegative numbers $\left(\gamma_{k}\right)_{k \geq 0}$ such that $\gamma_{k}=O^{\prime}\left(1 / 2^{k}\right)$, and for any $n \geq 0$ and $t \geq 0$,

$$
\lambda\left(E_{n} \cap E_{n+t}\right) \leq \lambda\left(E_{n}\right) \lambda\left(E_{n+t}\right)+\left(\lambda\left(E_{n}\right)+\lambda\left(E_{n+t}\right)\right) \gamma_{t}+\lambda\left(E_{n+t}\right) \gamma_{n} .
$$

However, using only $(\beta)$, we have

Corollary 4.1. For $\lambda$-a.e. $x \in\left[0,1\left[\right.\right.$, the sequence $\left(t_{n}(x)\right)_{n \geq 0}$ is uniformly distributed in $[0,1]$ and for all $\varepsilon>0, d \in[0,1]$, and $N \in \mathbb{N}^{*}$,

$$
A(N, x, d):=\#\left\{0 \leq n<N: 0 \leq t_{n}(x)<d\right\}=N d+O\left(\sqrt{N}(\log N)^{3 / 2+\varepsilon}\right) .
$$


P r o of. A straightforward computation gives

$$
\int_{0}^{1}\left|\sum_{n=M+1}^{M+N}\left(\mathbf{1}_{[0, d[}\left(t_{n}(x)\right)-d\right)\right|^{2} \lambda(d x)=O(N),
$$

and the corollary results from [Ga-Ko].

Remark 4.1. In a forthcoming paper with A. Thomas, we shall give, as an application, an alternative proof of this fact ([La-Th]). However, the present proof has the advantage that it presents materials that can be quite directly used for proving the nonindependence, or stochasticity, of the sequence $\left(t_{n}(\cdot)\right)_{n \geq 0}$.

\section{References}

[Eng] F. Engel, Entwicklung der Zahlen nach Stammbrüchen, in: Verhandlungen des 52sten Versammlung deutscher Philologen und Schulmänner in Marburg vom 29. September bis 3. October 1913, Leipzig 1914, 190-191.

[Esc] E. B. Escott, Rapid method for extracting a square root, Amer. Math. Monthly 44 (1937), 644-646.

[Ga-Ko] I. S. Gál et J. F. Koksma, Sur l'ordre de grandeur des fonctions sommables, C. R. Acad. Sci. Paris 227 (1948), 1321-1325.

[Gal] J. Galambos, Representations of Real Numbers by Infinite Series, Lecture Notes in Math. 502, Springer, 1976.

[Go-Sm] C. Goldie and R. L. Smith, On the denominators in Sylvester's series, Proc. London Math. Soc. (3) 54 (1987), 445-476.

[Khi] A. Ya. Khinchin, Continued Fractions, 3rd ed., Phoenix Books, The University of Chicago Press, 1935.

[Kn-Kn] A. Knopfmacher and J. Knopfmacher, A new infinite product representation for real numbers, Monatsh. Math. 104 (1987), 29-44.

[Ku-Ni] L. Kuipers and H. Niederreiter, Uniform Distribution of Sequences, Pure and Appl. Math., Wiley Interscience Series of Texts, Monographs, and Tracts, 1974.

[La-Th] Y. Lacroix and A. Thomas, Number systems and repartition mod 1, J. Number Theory, to appear.

[MF-VP] M. Mendès France and A. J. van der Poorten, From geometry to Euler identities, Theoret. Comput. Sci. 65 (1989), 213-220.

[Opp] A. Oppenheim, On the representation of real numbers by products of rational numbers, Quart. J. Math. Oxford Ser. (2) 4 (1953), 303-307.

[Ost] A. Ostrowski, Über einige Verallgemeinerungen des Eulerschen Produktes $\prod_{\nu=0}^{\infty}\left(1+x^{2^{\nu}}\right)=1 /(1-x)$, Verh. Naturforsch. Ges. Basel 11 (1929), 153-214.

[Per] O. Perron, Irrazionalzahlen, Chelsea, New York 1948.

[Pet] K. Petersen, Ergodic Theory, Cambridge Stud. Adv. Math. 2, Cambridge University Press, 1983.

[Phi] W. Philipp, Some metrical theorems in number theory, Pacific J. Math. 20 (1967), 109-127.

[Sch] F. Schweiger, Ergodic properties of fibered systems, draft version, Institut für Math. der Universität Salzburg, 1989. 
[Sch-1] F. Schweiger, Metrische Sätze über Oppenheimentwicklungen, J. Reine Angew. Math. 254 (1972), 152-159.

[Sie-1] W. Sierpiński, On certain expansions of real numbers into infinite fast converging products, Prace Mat. 2 (1958), 131-138 (in Polish; Russian and English summaries).

[Sie-2] —, Généralisation d'une formule de E. B. Escott pour les racines carrées, Bull. Soc. Roy. Sci. Liège 22 (1953), 520-529.

[Sta] P. Stambul, private communication.

[Ver] W. Vervaat, Success Epochs in Bernoulli Trials (with Applications in Number Theory), Math. Center Tracts 42, Mathematisch Centrum, Amsterdam 1972.

4, MONTÉE DE L'ANE CULOTTE TERRASSES DES OLIVIERS

13800 ISTRES, FRANCE

Received on 27.2.1992

and in revised form on 3.6.1992 